\title{
The efficient use of perimetry for neuro-ophthalmic diagnosis
}

University Eye Clinic, Vienna, Austria A Reitner

M Tittl

E Ergun

R Baradaran-Dilmaghani

Correspondence to: A Reitner, MD, University Eye Clinic, Dept A, Waehringer Guertel 1820, A-1090 Vienna, Austria.

\author{
A Reitner, M Tittl, E Ergun, R Baradaran-Dilmaghani
}

\begin{abstract}
Aims/background-Automated perimetry is both one of the most important diagnostic procedures in ophthalmology and the most difficult for the patient who is required to comply with strict and tiring conditions. This paper examines the use of a moving fixation target and a strategy for full threshold determination only in those data points found to be abnormal.

Methods-142 eyes in 71 patients were subjected to two types of visual field tests: the Dicon TKS 4000 Autoperimeter program 5 and the Humphrey field analyser program 30/2. The first procedure was compared with a commonly used instrument and strategy that is usually employed for the management of glaucoma patients.

Results-Such a strategy was used in patients presenting with neuroophthalmic problems taking an average testing time of 14.9 minutes. The alternative test described here took an average of 3.9 minutes, without loss of diagnostic value.

Conclusion-The shorter testing time results in greater patient acceptance, fewer fatigue induced artefacts, and the possibility of completing a perimetry test with patients whose ability might seriously be challenged by a longer test. The reduced time required and the more natural condition of not having to fixate on an unmoving position were found not to reduce the diagnostic value of the data produced in patients presenting for various reasons at a neuro-ophthalmology clinic.

(Br F Ophthalmol 1996;80:903-905)
\end{abstract}

The visual field screening of patients presenting at a busy neuro-ophthalmic department has different requirements from perimetry carried out for the purpose of glaucoma follow up; nevertheless, the same strategies are often used. In the management of patients with glaucoma the primary requirement is to be able to find and assess small progressions in a known field defect, eye by eye, with individuals who become experienced with perimetry techniques.

In the diagnosis of neurological diseases, perimetry is required to find and define unknown defects in the visual field of both eyes at the same session. Moreover, these patients are more likely to have attention problems and find perimetry a new difficult experience.
However, there is also the possibility that such patients also suffer from undiagnosed glaucoma.

In the neurological screening situation time is of the essence in two ways: firstly, there is no opportunity to perform four or five repeat tests in order to train ${ }^{1}$ the patient to be accurate as is needed in glaucoma management perimetry; secondly, the character of the patient population dictates that the test must be as quick and as stress free as possible.

This paper describes the results obtained from fields performed on 142 eyes in 71 perimetrically naive patients, comparing a conventional full threshold strategy, as commonly used both in glaucoma management and for all other perimetry purposes, with one that was thought to be less stressful. Both testing methods cover the central field and the peripheral field to 30 degree eccentricity.

\section{Patients and methods}

PATIENTS

A total of 142 eyes in 71 patients (females 47, males 24, average age 41.8 (SD14.9) years) were subjected to two types of visual field tests; the order in which each type of test was performed was randomised. The distribution of presenting reasons showed the typical variance of a neuro-ophthalmic outpatient department. Most of the patients participating this study were referred because of intracerebral tumour, papillary symptoms, macular symptoms, headache of unkown origin, or transient visual loss (Table 1 ).

INSTRUMENTS AND STRATEGIES USED

The standard reference test was carried out with a Humphrey autoperimeter using program 30/2. This thresholds 76 points in a square grid pattern within 30 degrees of eccentricity. The fixation point is unmoving, the background illumination is set at $10 \mathrm{~cd} / \mathrm{m}^{2}$, stimulus duration is $200 \mathrm{~ms}$, and interstimulus interval $200-400 \mathrm{~ms}$. The tests under investigation were carried out using a Dicon TKS

Table 1 Distribution (\%) of reasons for referral

Tumour

Cerebral vascular accident

Papillary symptoms

Macular symptoms

Headache of unknown origin

Optic neuritis

Transient visual loss

Aneurysm

Epilepsy

Siccadian syndrome

Keratoconus

Normal patients 
4000 perimeter using program 5 . This tests 80 points within 30 degrees of eccentricity in a radial pattern using a multi point suprathreshold screening strategy, ${ }^{2}{ }^{3}$ changing to single point full threshold determination in abnormal locations. The background illumination, dynamic range, and interstimulus interval were the same as with the Humphrey perimeter, but the stimulus duration was slightly longer at 250 $\mathrm{ms}$. The main visible difference to the patient was the moving fixation target. ${ }^{4}$

\section{Results}

The test variables checked from both perimeters showed a marked difference in testing time of 11.0 minutes ( Dicon program 5: 3.9 minutes/Humphrey program 30/2: $14.9 \mathrm{~min}-$ utes). Fixation losses, false positive responses, and false negative responses were obtained automatically in both strategies. After careful instruction before starting any of the strategies we found an acceptable compliance in all our patients (Table 2).

Of the 142 eyes, identical field results with both strategies were found in 57 eyes only (Table 3). Twenty five eyes showed field defects which were different in both tests. Of the 142 eyes, $9.8 \%$ had a ring defect in the Humphrey perimeter and an enlarged blind spot in the Dicon perimeter and $7.7 \%$ had an upper outer defect in the Humphrey and an enlarged blind spot in the Dicon perimeter (Figs 1 and 2). Of the 142 eyes, 42 had defects shown by the

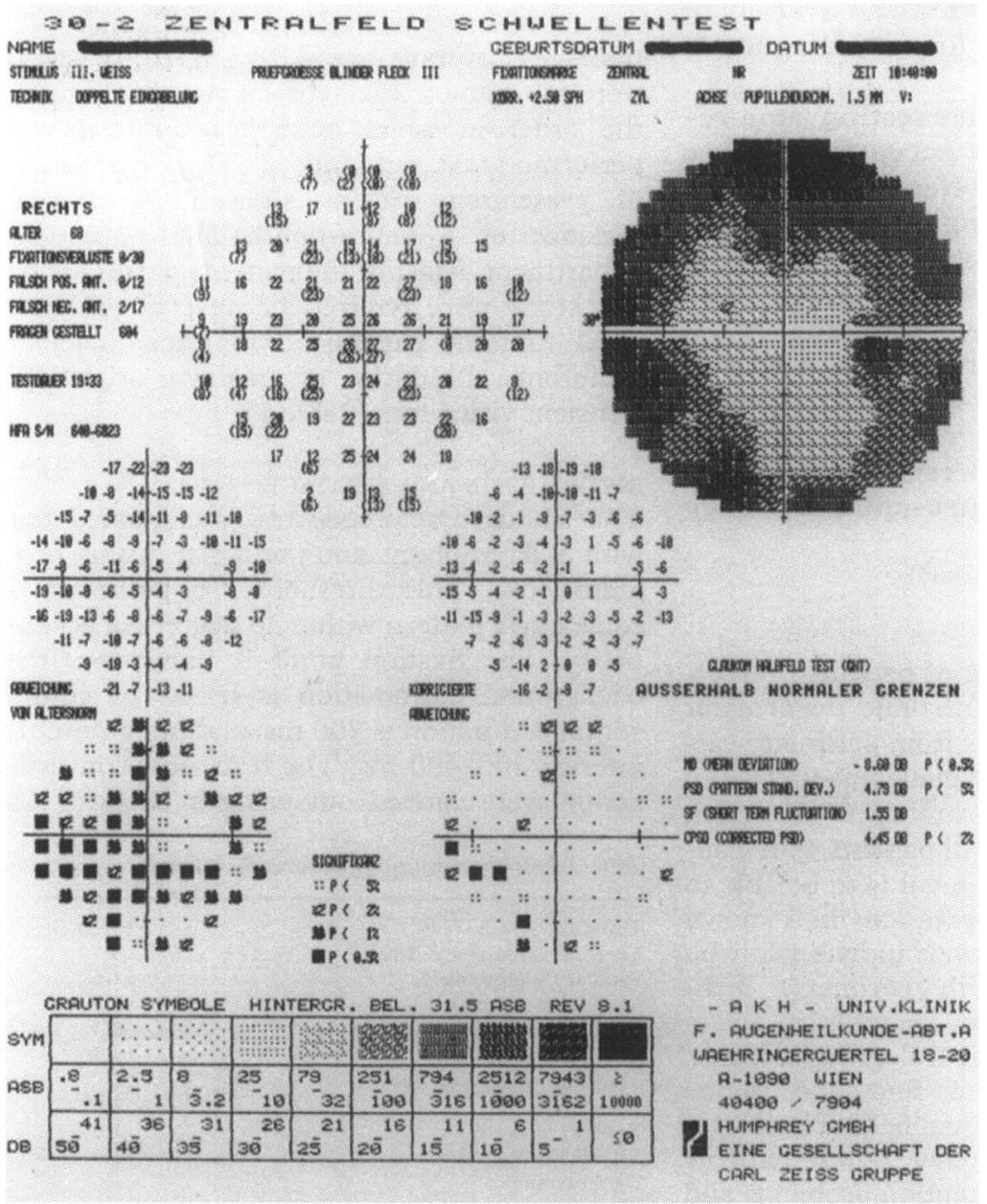

Figure 1 Typical artefact in Humphrey 30/2 perimetry.
Table 2 Test time and compliance related test variables recorded (SD) using Dicon and Humphrey programs

$\begin{array}{cl}\text { Test variables for Dicon program 5: } & \\ \text { Average test time (per eye) } & 3.9 \text { minutes } \\ \text { Fixation losses per minute } & 0.05(0.2) \\ \text { False positive responses } & 1.95(2.3) \\ \text { (Number of tests per field) } & 5.9(3.4) \\ \text { False negative responses } & 0 \\ \text { (Number of tests per field) } & 5.9(3.4) \\ \text { Test variables for Humphrey program } & 30 / 2: \\ \text { Average test time (per eye) } & 14.9 \text { minutes } \\ \text { Fixation losses per minute } & 0.0048(0.11) \\ \text { False posive responses } & 0.264(0.74) \\ \text { (Number of tests per field) } & 15.4(4.6) \\ \text { False negative responses } & 1.3(0.74) \\ \text { (Number of tests per field) } & 14.0(5.2)\end{array}$

$\star$ False responses were made without formal stimulus (such as during an interstimulus interval) or excess number of responses during a multistimulus presentation as a 'false positive'. Thus, the whole test procedure is a check for false positives.

Table 3 Distribution of identical field results (\%) with Dicon and Humphrey perimetry

\begin{tabular}{ll}
\hline Normal fields & 31.5 \\
Checkerboard scotomata & 5.2 \\
Relative scotoma or enlarged blind spot & 19.2 \\
Concentric ring scotomata & 10.52 \\
Central scotomata & 7.01 \\
Quadrant defects & 10.5 \\
Arcuate scotomata & 7.01 \\
Hemianopsia & 7.01 \\
\hline
\end{tabular}

Table 4 Comparison of defects (\%) identified by Humphrey and Dicon perimetry

Defects shown by Humphrey, Dicon

normal:

$\begin{array}{cl}\text { Ring defects } & 9.8 \\ \text { Defects close to upper 30 degrees } & 15.4 \\ \text { Unspecified relative scotomata } & 2.1 \\ \text { Lower quadrant defects } & 4.7 \\ \text { Defects shown by Dicon, Humphrey } & \\ \text { normal: } & \\ \text { Enlarged blind spot } & 8.4 \\ \text { Upper arcuate scotomata } & 0.7 \\ \text { Central scotomata } & 2.1 \\ \text { Temporal constriction } & 0.7 \\ \text { Unspecified relative scotomata } & 0.7\end{array}$

Humphrey but were normal in the Dicon perimeter and 18 eyes had defects shown by the Dicon but were normal in the Humphrey perimeter (Table 4). All fields with suspected artefacts were retested. ${ }^{4}$ After elimination of inconsistent artefacts by retesting, different field defects were found in only six eyes by the Dicon perimeter and five by the Humphrey perimeter.

\section{Discussion}

Ring defects are often caused by a refractive or fatigue related problem; the upper defect is often found to be an artefact due to drooping eyelids. Missed points near the blind spot may be caused by the radial test pattern being more dense and closer to the edge of the blind spot at this region; thus, small movements of the head can edge a stimulus into the blind spot area. The denser central distribution in this radial pattern may show small central defects more easily than a square grid as used by the Humphrey perimeter; this pattern should logically be better at defining arcuate defects, but the authors are unaware of any published proof of this.

It should be noted that, in addition to the increased test location density in the Dicon 80 point radial pattern near the blind spot, the 


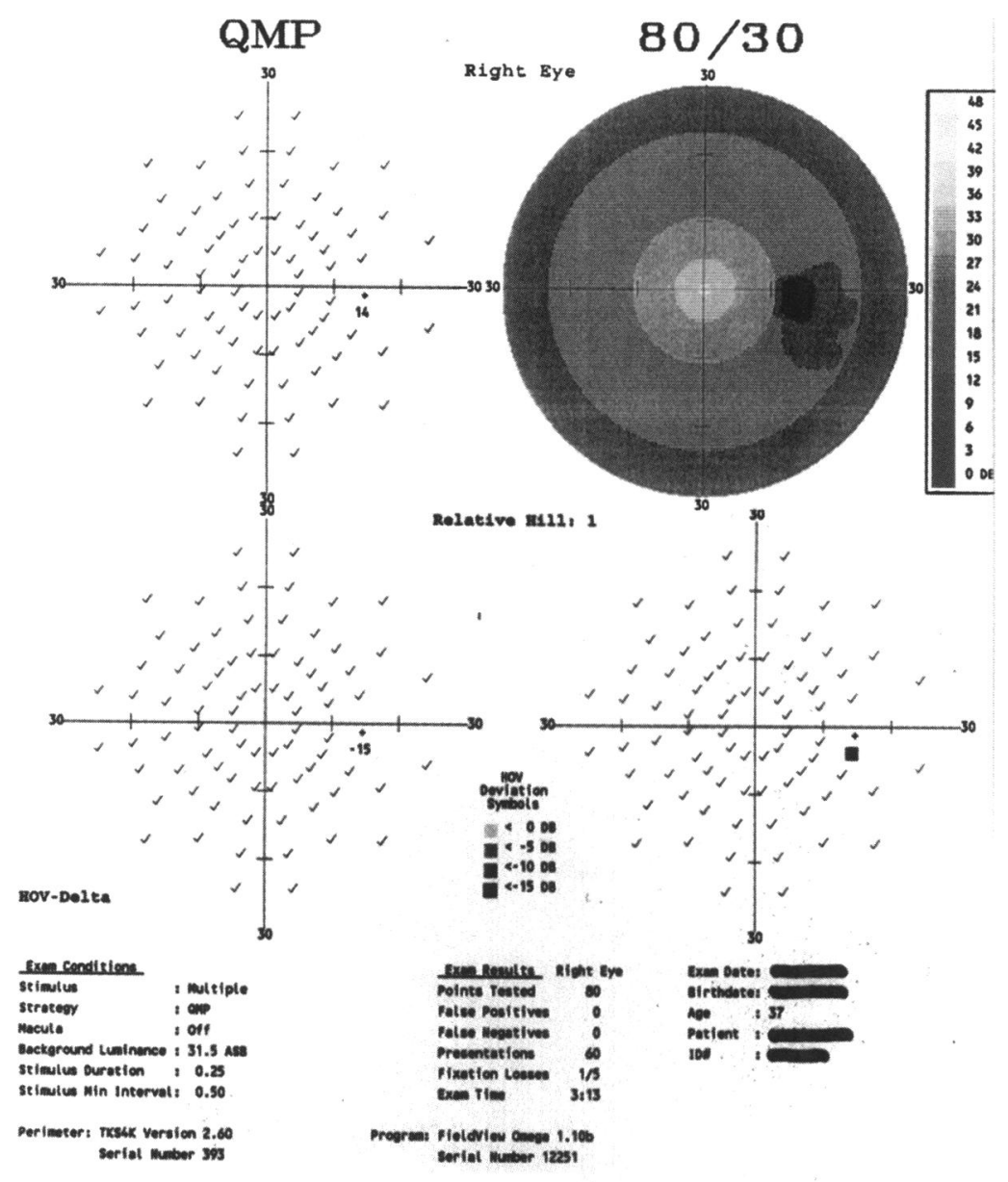

DICON•

Figure 2 Typical artefact in Dicon program 5 perimetry.

occasional missed point next to the blind spot is not normally regarded as a pathological abnormality. Allowing for this, the defects found by the Dicon perimeter but not by the reference procedure on the Humphrey perimeter are: one case of temporal constriction, one of unspecific relative scotoma, one of arcuate scotoma, and three small central scotomata. It is interesting that a procedure thought to be advantageous for screening neurological patients seems to be successful in finding small scotomata of a type more associated with glaucoma; perhaps because of the use of a radial pattern rather than a square grid that makes the mathematics of programming for grey scale displays simpler.
The artefacts on first testing found on the Humphrey perimeter, which can be attributed to fatigue causing drooping eyelids or attention contraction, were found in 57 eyes or $40 \%$ of the total, which is not surprising considering the increase in testing time.

The differences in false positive responses (positive or negative) reflect the differences in testing conditions and the different criteria used in each strategy. In the case of the Dicon perimeter the stimulus to respond after each movement of the fixation target is countered by target movements finishing without a stimulus followed by astonishment if the patient gives false response. Other authors ${ }^{5}$ have not found this to be a problem. Moreover, in comparing the number of false positives recorded by the two systems throughout the test (not just those that occur in response to a specific trial as in the Humphrey perimeter) the major difference was lack of false negatives with the Dicon perimeter, which can be attributed to the shorter testing time, or the more attention holding moving fixation target, or to a combination of both of these. ${ }^{6}$

\section{Conclusion}

After the elimination of known artefacts caused by fatigue in long periods of perimetry or by the test pattern density near the blind spot, there was little difference in the clinical usefulness of the compared strategies.

However, the striking difference was the reduced testing time with the Dicon program 5 by a factor of 3.8 ; this is only $26 \%$ of the time necessary for the Humphrey perimeter program 30/2. This shorter testing time can result in less patient stress, less patient aversion to repeat perimetry, fewer fatigue induced artefacts, and higher patient throughput in a given time.

1 Wood JM, Wild JM, Hussey MK. Serial examination of the normal visual field using Octopus automated projection perimetry. Evidence for a learning effect. Acta Ophthalmol 1987;65:326-33.

2 Jacobs NA, Patterson $\mathrm{IH}$. Variability of the hill of vision and its significance in automated perimetry. $\mathrm{Br} \mathcal{F}$ Ophthalmol 1985;69:824-6.

3 Henson DB, Anderson R. Thresholds using single and multiple stimulus presentations. Perimetry Update 1988/89; 191-6.

$4 \mathrm{Yi} \mathrm{Li}$, Mills RP. Kinetic fixation improves threshold sensitivity in the central visual field. F Glaucoma 1992;1:108-16.

5 Chauhan BC, McCormick TA, Whelan JH, Mohandas RN. Variability of normal visual fields in a prospective study. Perimetry Update 1994/95;27-30.

6 Demirel S, Vingrys AJ. Acceptable false response rates for reliable perimetric outcomes. Perimetry Update 1994/95; 